\title{
Self-efficacy to Engage in Physical Activity and Overcome Barriers, Sedentary Behavior, and Their Relation to Body Mass Index Among Elderly Indonesians With Diabetes
}

\author{
Qonita Rachmah', Stefania Widya Setyaningtyas', Mahmud Aditya Rifqi', Diah Indriani ${ }^{2}$, Triska Susila Nindya', \\ Hario Megatsari', Trias Mahmudiono', Wantanee Kriengsinyos ${ }^{4}$ \\ ${ }^{1}$ Department of Nutrition, Faculty of Public Health, Universitas Airlangga, Surabaya, Indonesia; ${ }^{2}$ Department of Biostatistics, Faculty of Public \\ Health, Universitas Airlangga, Surabaya, Indonesia; ${ }^{3}$ Department of Health Promotion, Faculty of Public Health, Universitas Airlangga, Surabaya, \\ Indonesia; ${ }^{4}$ Nutrition and Dietetics Program, Institute of Nutrition, Mahidol University, Salaya-Nakhon Pathom, Thailand
}

Objectives: Elderly individuals with diabetes should maintain a normal body mass index (BMI) to help control their blood glucose levels. This study investigated barriers to physical activity (PA), self-efficacy to overcome those barriers, and PA self-efficacy among elderly individuals with diabetes in relation to BMI.

Methods: This cross-sectional study included 56 participants. Data were collected by a questionnaire interview and direct measurements for anthropometric data. PA self-efficacy was measured using 8 questions describing different levels of PA, where participants rated the strength of their belief that they could engage in that activity. Self-efficacy to overcome barriers was measured using 10 questions capturing participants' confidence in their ability to engage in PA despite different possible barriers. Mean scores for these parameters were analyzed using the chi-square test and the independent $t$-test.

Results: In total, $89.3 \%$ of participants had a low PA level and $58.9 \%$ had more than 3 hours of sedentary activity per day. Furthermore, $55.4 \%$ were obese and $14.3 \%$ were overweight. The mean scores for PA self-efficacy and self-efficacy to overcome barriers were $59.1 \pm$ 26.4 and $52.5 \pm 13.8$, respectively. PA level was related to BMI $(p<0.001 ; r=0.116)$ and sedentary activity $(p<0.05 ; r=0.274)$. PA self-efficacy and age were not related to BMI. Barriers to PA were associated with PA levels $(p<0.05)$.

Conclusions: Physical inactivity was a major problem in elderly individuals with diabetes, and was correlated with higher BMI. Lower levels of PA might be mediated by sedentary activity.

Key words: Body mass index, Barriers, Diabetic elderly, Physical activity, Self-efficacy, Indonesia

\section{INTRODUCTION}

Received: January 3, 2019 Accepted: June 13, 2019

Corresponding author: Qonita Rachmah, MSc Department of Nutrition, Faculty of Public Health, Universitas

Airlangga, Surabaya 60111, Indonesia

E-mail: qonita.rachmah@fkm.unair.ac.id

This is an Open Access article distributed under the terms of the Creative Commons Attribution Non-Commercial License (http://creativecommons.org/licenses/by$\mathrm{nc} / 4.0 /$ ) which permits unrestricted non-commercial use, distribution, and reproduction in any medium, provided the original work is properly cited.
The elderly often experience various health problems, such as osteoporosis, type 2 diabetes mellitus (DM), hypercholesterolemia, hypertension, and other cardiovascular diseases, which negatively impact quality of life and pose a risk for mortality [1]. The burden of these conditions has been exacerbated by the increase in the elderly population as a result of demographic changes. In Indonesia, the elderly population is 18.781 million and is predicted to reach 36 million in 2025 
$[2,3]$. A national survey found that 6 of 10 deaths in Indonesia were caused by degenerative diseases, and that $50 \%$ of those deaths were caused by cardiovascular diseases. DM is a degenerative disease with a significant worldwide burden, as it increases the risk of premature death by 5 -fold, that of death from heart disease 4-fold, and that of death from kidney disease 20-fold, to the point that is responsible for one-third of all deaths [1]. Furthermore, the prevalence of DM is higher among the elderly. For example, in Thailand its prevalence has reached $8 \%$ in those over 50 years of age [4], while in Indonesia, approximately $50 \%$ of patients with diabetes are aged $>65$ years [5], with a prevalence of 5.5\% among those aged 55-64 years [6].

Obesity is well known to be a major risk factor for type 2 DM, as many studies have confirmed the link of overweight and obesity with diabetes. For instance, a 14-year follow-up analysis in the nurses' health study revealed that women with a baseline body mass index (BMI) $>35.0 \mathrm{~kg} / \mathrm{m}^{2}$ had a 49 times higher risk of developing diabetes than those with a baseline BMI less than $22.0 \mathrm{~kg} / \mathrm{m}^{2}$ [7]. Elevated BMI, as a marker of overweight and obesity, mainly occurs due to an energy imbalance in which energy intake from food is higher than energy expenditure. Low levels of energy expenditure can be caused by lower levels of physical activity (PA) or higher levels of sedentary activity. The most recent national survey in Indonesia revealed a high proportion of sedentary activity among the elderly, of whom at least $66.9 \%$ were physically inactive [6]. Lifestyle changes have been proven to be a viable strategy for managing type 2 DM. A meta-analysis of elderly Southeast Asians showed that dietary and PA modifications, such as gymnastics, tai chi, or yoga exercises, could reduce blood sugar and hemoglobin A1c levels in patients with diabetes. However, not all elderly individuals with diabetes are able to change their behavior in ways that promote health [8].

Based on social cognitive theory, a person can change his or her behavior through an increase in self-efficacy or self-confidence in the capability to do so [9]. Self-efficacy is not itself a barrier, since it affects factors that can act as barriers, such as outcome expectations or socio-structural factors. High levels of self-efficacy can lead to healthy behaviors. In addition, according to the Centers for Disease Control and Prevention, the most common barriers to PA in adults include a lack of time, low self-motivation, the feeling that it is inconvenient, the feeling that PA is not enjoyable or boring, fears of being injured, lack of support from family and friends, and unfriendly environments [10].
In patients with diabetes, maintaining a normal BMI is crucial for managing diabetes, and in particular for preventing increases in blood glucose levels. A study comparing insulin sensitivity across all age and weight categories (normal to obese) found that insulin resistance was closely associated with physical inactivity and obesity, rather than being an effect of aging [11]. Those studies strengthen the hypothesis that controlling obesity by increasing PA could help maintain insulin sensitivity, thereby improving the management of diabetes. This study was conducted with the goal of understanding barriers to PA, self-efficacy to overcome barriers, and self-efficacy to engage in PA among elderly individuals with diabetes in relation to $\mathrm{BMI}$, as an indicator of obesity. Understanding the level of selfefficacy of elderly individuals with diabetes through this baseline study could assist in the development of specific interventions to help them make PA part of their daily routine.

\section{METHODS}

This was a descriptive analytic study using a cross-sectional design. The sample size was calculated using the test for difference in 2 independent proportions, and the minimum sample size required was 50 . We therefore enrolled 56 participants, anticipating a $10 \%$ dropout rate. Based on the results of the study, the statistical power was calculated to be 1.0. Participants in this baseline study were identified using multistage random sampling from the database of the local public health center (Jagir public health center). Before participants were enrolled in the study, several inclusion and exclusion criteria were applied. The inclusion criteria were being Indonesian, having diabetes, being $>55$ years of age, being able to read and write in Bahasa Indonesia, being able to live independently, and engaging in normal activities of daily living. Elderly individuals who had an illness preventing engagement in normal activities of daily living at the time of the study were excluded. Anthropometric data (weight and height) were collected through direct measurements made with a digital scale with $0.1 \mathrm{~kg}$ precision (Body Impedance Analyzer, Omron HBF375 , Kusatsu, Japan) and a stadiometer with $0.1 \mathrm{~cm}$ precision (Seca GmbH, Hamburg, Germany). Social and demographic data (age, sex, education level, work status, family type, and number of children) were collected using a questionnaire. PA level and sedentary behavior data were collected using the validated Global Physical Activity Questionnaire (GPAQ), which is also used in national surveys and consists of 4 main sections: 
working activity, traveling activity, recreational activity, and sedentary activity $[6,12]$. Data for age and number of children are presented as mean \pm standard deviation, minimum, and maximum. Participants were categorized by sex as male or female, education level was categorized into 4 categories (not having attended school, primary school, secondary school, and university), work status was dichotomized as working or not working at present, and family type was categorized as nuclear or batih (extended). Based on the GPAQ, PA as classified as light, moderate, or vigorous based on metabolic equivalents (METs) per week; furthermore, PA was dichotomized as moderate to vigorous for values of $\geq 600 \mathrm{MET} / \mathrm{wk}$ and light for values of $<600 \mathrm{MET} / \mathrm{wk}$ [13].

Data on self-efficacy to overcome barriers to PA and PA selfefficacy were gathered using a structured questionnaire. Selfefficacy to overcome barriers to PA was measured using 10 questions that presented several barriers, while PA self-efficacy was measured using 8 questions describing different levels of PA and requesting subjects to rate the strength of their belief that they could engage in that activity. The validity of the questionnaire was evaluated using the Cronbach $\alpha$, and moderate reliability was found for both questionnaires $(0.682$ and 0.576 , respectively). The 10 questions assessing self-efficacy to overcome barriers requested participants to rate their confidence in performing PA in the following circumstances: (1) feeling tired, (2) poor weather conditions, (3) experiencing personal problems (or after doing so), (4) experiencing depression, (5) feeling anxious, (6) recovering from injuries or illness, (7) feeling uncomfortable while exercising or walking, (8) during or after the holidays, (9) having too much housework, and (10) there being another thing that is more interesting to do. The 8 questions on PA self-efficacy asked participants to assess their confidence to engage in walking (1) $1000 \mathrm{step} / \mathrm{d}$, (2) $2000 \mathrm{step} / \mathrm{d}$, (3) $3000 \mathrm{step} / \mathrm{d}$, (4) $4000 \mathrm{step} / \mathrm{d}$, (5) $6000 \mathrm{step} / \mathrm{d}$, (6) 8000 step/d, (7) 10000 step/d, and (8) 12000 step/d. Respondents rated their confidence in overcoming a barrier or engaging in a specific amount of PA on a scale from 0 to 100. The mean scores of self-efficacy to engage in PA and to overcome barriers were calculated for further analysis.

All respondents were interviewed by trained enumerators. Statistical analysis was performed using the chi-square test, Pearson correlation coefficients, and the independent $t$-test to analyze the associations between the independent variables and the dependent variable (BMI) with $95 \%$ confidence intervals (Cls) and significance at the 0.05 level (2-tailed). Then, lo- gistic regression was performed to estimate adjusted odds ratios (ORs), controlling for sex as a confounding factor.

\section{Ethics Statement}

This study was conducted according to the guidelines outlined in the Declaration of Helsinki, and all procedures involving human subjects were approved by the Institutional Review Board (IRB) of the Faculty of Public Health of Universitas Airlangga (IRB no. 414-KEPK). Written informed consent was obtained from the participants. Respondents were informed that they could withdraw their participation in the study at any time without consequences.

\section{RESULTS}

\section{Description of the Sample}

As seen in Table 1, most of the participants were female (71.4\%), and half had completed a primary education (mean-

Table 1. Characteristics of the elderly individuals with diabetes enrolled in this study

\begin{tabular}{lc}
\hline Characteristic & $\mathbf{n}(\%)$ \\
\hline Age, mean \pm SD [Min-Max] (y) & $66.8 \pm 5.3[56-80]$ \\
No. of children, mean \pm SD [Min-Max] (n) & $3.4 \pm 2.2[0-12]$ \\
Sex & \\
Male & $16(28.6)$ \\
Female & $40(71.4)$ \\
Education level & \\
No formal education & $3(5.4)$ \\
Primary school & $16(46.4)$ \\
Secondary school & $19(33.9)$ \\
University & $8(14.3)$ \\
Work Status & \\
Working & $10(17.8)$ \\
Not working & $46(82.2)$ \\
BMl category & \\
Underweight & $1(1.8)$ \\
Normal & $16(28.6)$ \\
Overweight & $8(14.3)$ \\
Obese & $31(55.4)$ \\
Physical activity level & \\
Moderate to vigorous & $6(10.7)$ \\
Light & $50(89.3)$ \\
Sedentary activity (hr/d) & \\
$>3$ & $33(58.9)$ \\
$\leq 3$ & $23(41.1)$ \\
\hline & \\
\hline & \\
\hline
\end{tabular}

SD, standard deviation; Min, minimum; Max, maximum; BMI, body mass index. ${ }^{*} p<0.05$ with BMI $(r=0.274)$. 
ing that they had finished elementary to junior high school), while only $14.3 \%$ had attended university. Only $17.8 \%$ of the elderly participants were still working, whereas the rest had retired already. In Indonesia, the average age of retirement is 55 years and the maximum is 60 years [14]. Working status was related to PA in the elderly participants, although we did not further examine this association according to occupation. In terms of family type, $70 \%$ of the participants lived with their nuclear family (husband, wife, and children), while 30\% lived in a batih (extended) family structure, including in-laws and grandchildren. The mean age of the participants was $66.8 \pm$ 5.3 years, which reflects the findings of a previous study in Indonesia that the prevalence of diabetes increases with age.

\section{Body Mass Index Distribution, Physical Activity Level, and Sedentary Activity Among the Participants}

Table 1 presents the distribution of BMI, PA levels, and sedentary activity among the participants. In accordance with the

Table 2. Self-efficacy to overcome barriers to PA and to engage in PA $(n=56)$

\begin{tabular}{lcc}
\hline Variable & Mean \pm SD & Min-Max \\
\hline Self-efficacy to overcome barriers & & \\
Feeling tired & $54.2 \pm 24.1$ & $0-100$ \\
Poor weather conditions & $54.4 \pm 20.3$ & $20-100$ \\
Experiencing personal problems & $53.4 \pm 21.3$ & $10-100$ \\
Experiencing depression & $49.1 \pm 21.1$ & $0-100$ \\
Feeling anxious & $49.9 \pm 21.1$ & $0-100$ \\
Recovering from injuries or illness & $49.7 \pm 18.9$ & $10-100$ \\
Feeling uncomfortable while & $48.3 \pm 18.3$ & $10-90$ \\
$\quad$ exercising/walking & & \\
During or after the holidays & $56.7 \pm 23.5$ & $20-100$ \\
Having too much housework & $52.8 \pm 24.2$ & $0-100$ \\
There being another thing that is & $56.5 \pm 23.2$ & $10-100$ \\
more interesting to do & & \\
Overall & $59.1 \pm 26.4$ & - \\
Self-efficacy to engage in PA (walking, step/d) & & \\
1000 & $83.2 \pm 21.8$ & $20-100$ \\
2000 & $75.4 \pm 28.3$ & $10-100$ \\
3000 & $71.3 \pm 29.1$ & $10-100$ \\
4000 & $62.9 \pm 30.9$ & $10-100$ \\
6000 & $55.7 \pm 33.8$ & $0-100$ \\
8000 & $48.9 \pm 34.6$ & $0-100$ \\
10000 & $42.5 \pm 32.7$ & $0-100$ \\
12000 & $33.2 \pm 31.8$ & $0-100$ \\
Overall & $52.5 \pm 13.8$ & - \\
\hline & & \\
\hline
\end{tabular}

PA, physical activity; SD, standard deviation; Min, minimum; Max, maximum. hypothesis that obesity is a major risk factor for diabetes, we found that $69.7 \%$ of the participants were overweight and obese. Light PA was more common among the participants $(89.3 \%)$ than moderate to vigorous activity $(10.7 \%)$, and PA was significantly associated with BMI $(p<0.05)$. Specifically, the participants with a moderate to vigorous PA level had a lower BMI than those who engaged in light PA $\left(25.06 \mathrm{~kg} / \mathrm{m}^{2}\right.$ vs. $24.78 \mathrm{~kg} / \mathrm{m}^{2}$ ). This result was also supported by the finding that a majority of the participants were sedentary for more than $3 \mathrm{hr} / \mathrm{d}$ (58.9\%), with a mean duration of sedentary activity of $4.2 \pm 3.8$ hours. Similarly to the PA level, sedentary activity was also related to $\mathrm{BMI}(p<0.05)$ in elderly individuals with diabetes.

\section{Self-efficacy to Engage in Physical Activity and to Overcome Barriers}

Self-efficacy to engage PA and to overcome barriers to PA were measured using a Likert scale ranging from 0 to 100 rating respondents' confidence to carry out specific tasks. We found that highest barrier for the elderly to engage in PA was feeling uncomfortable while exercising or walking, with a mean score of $48.3 \pm 18.3$, followed by experiencing depression ( $49.1 \pm$ 21.1), recovering from injuries or health problems (49.7 \pm 18.9$)$, feeling anxious (49.9 \pm 21.1 ), and excessive housework (52.8 \pm 24.2). Details on the scores for each question are presented in Table 2. The results for PA self-efficacy showed that the participants were confident about their ability to take 1000 steps in a day (83.2 \pm 21.8$)$, but their self-efficacy decreased for increasing

Table 3. Self-efficacy to overcome barriers to PA and selfefficacy to engage in PA in relation with BMI, PA level, and sedentary activity

\begin{tabular}{lcc}
\hline Variable & $\begin{array}{c}\text { Self-efficacy to } \\
\text { engage in PA }\end{array}$ & $\begin{array}{c}\text { Self-efficacy to } \\
\text { overcome barriers }\end{array}$ \\
\hline BMl & $55.7 \pm 28.1$ & $51.1 \pm 12.1$ \\
Normal & $60.6 \pm 25.9$ & $53.1 \pm 13.5$ \\
Overweight-obese & 0.072 & 0.436 \\
$p$-value & & \\
PA level & $64.4 \pm 21.6$ & $55.3 \pm 14.3$ \\
Moderate to vigorous & $58.5 \pm 27.1$ & $52.2 \pm 13.1$ \\
Light & 0.001 & 0.001 \\
$p$-value & & \\
Sedentary activity (hr/d) & $58.1 \pm 23.9$ & $52.2 \pm 12.8$ \\
$>3$ & $60.6 \pm 30.2$ & $52.9 \pm 12.4$ \\
$\leq 3$ & 0.002 & 0.064 \\
$p$-value & &
\end{tabular}

PA, physical activity; BMI, body mass index. 
Table 4. Results of the logistic regression models adjusted for sex

\begin{tabular}{|c|c|c|c|c|}
\hline Variable & $\mathbf{n}$ & Crude OR (95\% CI) & aOR (95\% CI) & $p$-value \\
\hline \multicolumn{5}{|l|}{ PA level } \\
\hline Self-efficacy to engage in PA & 56 & $0.86(0.72,0.96)$ & $1.03(1.03,1.96)$ & 0.013 \\
\hline Self-efficacy to overcome barriers & 56 & $0.92(0.86,0.98)$ & $0.96(0.94,0.99)$ & 0.004 \\
\hline \multicolumn{5}{|l|}{ Sedentary activity } \\
\hline Self-efficacy to engage in PA & 56 & $1.03(1.00,1.92)$ & $1.12(1.02,1.98)$ & $<0.001$ \\
\hline Self-efficacy to overcome barriers & 56 & $\mathrm{~N} / \mathrm{A}$ & $\mathrm{N} / \mathrm{A}$ & $\mathrm{N} / \mathrm{A}$ \\
\hline
\end{tabular}

OR, odds ratio; $\mathrm{Cl}$, confidence interval; aOR, adjusted odds ratio; PA, physical activity; N/A, not available.

daily step counts, as presented in Table 2.

In our analysis of these findings according to BMI (Table 3), we found that overweight/obese participants were more confident in their ability to engage in PA, with a higher self-efficacy score $(60.6 \pm 25.9)$ than those with a normal BMI (55.7 \pm 28.1). A similar result was found in self-efficacy to overcome barriers, with overweight/obese participants having better self-efficacy (53.1 \pm 13.5$)$ than those with a normal weight $(51.1 \pm 12.1)$. However, we did not find any significant differences. Furthermore, in general, we found that the self-efficacy of participants to engage in PA $(59.1 \pm 26.4)$ was higher than their self-efficacy to overcome barriers to engage in PA (52.5 \pm 13.8). Self-efficacy also showed a significant relationship with the PA level of the participants $(p=0.001)$. Only self-efficacy to engage in PA was significantly related to sedentary activity $(p=0.002)$, meaning that elderly individuals with diabetes who had better self-efficacy were more likely to spend $\leq 3$ hours of sedentary time in a day. Table 4 presents the results of binary logistic regression models adjusted for sex, which aimed to detect possible confounding in the study. The results showed that sex was not a confounder in the relationships of self-efficacy to engage in PA and to overcome barriers with PA level, or in the association between self-efficacy to engage in PA and sedentary activity.

\section{DISCUSSION}

The objective of this study was to identify barriers to PA and to characterize self-efficacy to overcome those barriers and to engage in PA among elderly individuals with diabetes in relation to BMI. We focused on elderly individuals with diabetes with the eventual goal of designing an intervention to assist in diabetes management and to prevent severe complications. First, most of our study participants were elderly females with diabetes, paralleling several other studies, such as a population-based study among Zuni Indians that found that diabetes prevalence was $57 \%$ higher in the female population than in the male population [15] and a case-control study that revealed that both post-prandial and fasting blood glucose levels were higher in females than in males [16]. A broader Indonesian national survey similarly found that the prevalence of diabetes was higher in females (2.3\%) than in males (2.0\%) [6]. Moreover, diabetes may decrease the lifespan of females by 8.2 years, in contrast to 7.5 years in males. The reason for this discrepancy may be that hormonal differences contribute to a higher diabetes risk in females, especially after the dramatic hormonal changes that take place during reproductive age and after menopause [17]. Other characteristics of respondents, such as education level, type of family, and age also contributed to diabetes management. Education level is closely related to knowledge, which could influence a person's behavior. However, many factors other than knowledge, such as self-efficacy, also contribute to behavior. When developing a strategy for controlling diabetes, education level should be considered along with other factors. Furthermore, households with more people have a greater need for food, which could affect the individual intake. Physiological changes in the body after age 30 could increase fasting blood sugar levels by 1-2 $\mathrm{mg} / \mathrm{dL} / \mathrm{y}$ and 3-hour post-prandial glucose levels by 5.6-13.0 $\mathrm{mg} / \mathrm{dL} / \mathrm{y}$ [18]. In addition, the risk of diabetes is sharply elevated in overweight individuals aged $>45$ years due to glucose intolerance and insufficient production of insulin by pancreatic $\beta$-cells. An analysis of data from the National Health and Nutrition Examination Survey (NHANES) 1999-2000 and the NHANES III 1988-1994 also found that the age of diabetes diagnosis has decreased over time, indicating a trend for diabetes to occur at earlier ages [19].

Our study revealed that 7 of 10 elderly individuals with diabetes were overweight or obese. This result corresponds to the findings of the FIBER study in Brazil, which observed that diabetes was correlated with central obesity, especially among females [20]. A long period of overweight and obesity induces 
the pathogenesis of type 2 diabetes. This can be explained by at least 2 mechanisms. The first mechanism is that the accumulation of adipose tissue, specifically visceral, induces a spectrum of metabolic and hormonal changes, which progressively impair the insulin signal transduction pathway and manifest as increasing insulin resistance in adipose tissue, liver, and skeletal muscle. The second possible mechanism through which obesity worsens the pathogenesis of diabetes is altered adipokine production and an increased inflammatory load as obesity becomes more severe [21]. The higher risk of diabetes in the elderly was also explained to be related to age-related changes in carbohydrate metabolism, such as insulin-mediated glucose disposal resistance and shifts in glucose-induced insulin release [22].

In our study, elderly individuals with diabetes who engaged in only light PA had a mean BMl that was higher by $0.274 \mathrm{~kg} / \mathrm{m}^{2}$. A randomized clinical trial of a minimum of 150 minutes of brisk walking or similar-intensity PA and 7\% weight loss among 3234 individuals with impaired glucose tolerance resulted in a $58 \%$ reduction of diabetes incidence [23]. This strengthens the proposal that PA together with weight management could improve diabetes control. Beyond its relationship with BMI, PA should be increased among type 2 diabetes patients due to its impact on several outcomes. Physically inactive adults with type 2 diabetes have a higher risk for cardiovascular disease mortality [24], as well as a lower life expectancy, poorer control of blood glucose, and poorer status regarding risk factors for coronary artery disease [25-27]. Our results regarding sedentary activity were more favorable than those of a national survey in Indonesia reporting that $65.6 \%$ of older adults had $>3$ hours of sedentary activity in a day [6]. Higher levels of sedentary activity lead to a decline in energy expenditure, which could lead to fat buildup in adipose tissue. Reduction of sedentary activity to $<3 \mathrm{hr} / \mathrm{d}$ may increase life expectancy by 2 years [28].

This study also found that elderly individuals with diabetes had high mean scores for PA self-efficacy, meaning that they were confident in their ability to perform the PAs presented in the questionnaire. An interventional study in older adults after cardiac events suggested that self-efficacy in terms of expectations for PA was significantly correlated with distance in a walking intervention [29]. However, the barriers presented in the questionnaire might not have been relevant for each participant, leading to variation in the results. Feeling uncomfortable while exercising or walking was the most common barrier reported by the participants. Based on interviews during data collection, most of the elderly found that their environment did not support their engagement in PA due to factors such as narrow roads, the presence of many vehicles, and insufficient space to engage in proper exercise. Therefore, they did not feel comfortable. We suggest that proper spaces, such as parks, should be constructed for the elderly to engage in PA. Other barriers for elderly individuals with diabetes to engage in PA were experiencing depression, recovering from injuries or health problems, feeling anxious, and excessive housework. Furthermore, a study among older adults with different socioeconomic status explained that more barriers were experienced by older adults with low socioeconomic status due to poor knowledge of PA guidelines, neighborhood safety, and health conditions [30].

However, neither self-efficacy to engage in PA nor self-efficacy to overcome barriers was associated with the BMI of the participants, although significant associations were found with PA level (Table 4). Participants who engaged in moderate to vigorous PA had higher PA self-efficacy and higher self-efficacy to overcome barriers than their counterparts who engaged in light PA. As a pilot study, this result implies that enhancing self-efficacy may be a promising way to increase PA among the elderly. Guicciardi et al. [31] demonstrated that PA to reduce the morbidity of diabetes requires changes lasting a lifetime; thus, it is especially important to target readiness to change behavior. Furthermore, they explained that self-efficacy to engage in exercise increases the willingness to change, from pre-contemplation to the maintenance stage, based on the trans-theoretical model.

Only PA self-efficacy showed a significant correlation with sedentary activity, as participants who engaged in less sedentary activity ( $\leq 3 \mathrm{hr} / \mathrm{d}$ ) were more confident in their ability to engage in PA than those with $>3$ hours of sedentary activity per day. In this study, the following barriers to PA were found among elderly individuals with diabetes: not feeling comfortable doing PA, recovering from health problems, anxiety, excessive housework, and personal problems (feeling lonely). These findings could be used as the basis for interventions related to diabetes management. Bethancourt et al. [32] conducted a qualitative study exploring several ways to overcome barriers to PA among older adults; for factors in the physical environment that cause discomfort, options include encouraging PA at home and building a convenient or nearby location for PA or walking. Issues relating to individual preferences 
or mental health problems can be overcome by raising awareness of the benefits of PA, providing encouragement, promoting the proactive pursuit of programs, and combining PA with enjoyable or daily activities. Encouragement from peers, companionship, guidance from professionals, social contacts, or using role models of healthy and active elderly individuals are also options for overcoming barriers.

In conclusion, this study revealed that a lower PA level was associated with PA self-efficacy and self-efficacy to overcome barriers to engage in PA. These factors did not show a direct relationship with BMI, but BMI was associated with PA level. The strengths of this study include the reliability and validity of the questionnaire used; furthermore, the results provided a useful overview of several barriers among elderly individuals with diabetes and will be further used for develop an intervention related to increasing self-efficacy or reducing barriers. Additionally, lower levels of PA might be mediated by higher levels of sedentary activity among elderly individuals with diabetes. Efforts to improve PA among elderly individuals with diabetes should focus on improving self-efficacy and overcoming barriers, as well as BMI. However, this study had several limitations, including a low sample size, which means that the results of this study might not be generalizable to the entire population, and the use of BMI as an indicator of obesity, although BMI might not be representative for the older population. Other limitations of the study are that most respondents had a low level of PA (89.3\%) and that bias due to the self-reporting methodology might have been present.

\section{CONFLICT OF INTEREST}

The authors have no conflicts of interest associated with the material presented in this paper.

\section{ACKNOWLEDGEMENTS}

We would like to thank the Faculty of Public Health of Universitas Airlangga for funding this research, as well as the enumerators and participants of the study.

\section{AUTHOR CONTRIBUTIONS}

Conceptualization: QR, TM, SWS, WK, MAR, HM, TSN, DI. Data curation: MAR, WK. Formal analysis: DI, HM. Funding acquisition: TSN, QR. Methodology: TM, QR. Project administration:
SWS. Visualization: SWS, MAR, QR. Writing - original draft: QR. Writing - review \& editing: QR, TM, SWS, WK, MAR, HM, TSN, DI.

\section{ORCID}

Qonita Rachmah https://orcid.org/0000-0002-6094-4885

Stefania Widya Setyaningtyas https://orcid.org/0000-00022331-2037

Mahmud Aditya Rifqi https://orcid.org/0000-0003-49536159

Diah Indriani https://orcid.org/0000-0002-7584-2910

Triska Susila Nindya https://orcid.org/0000-0003-0870-5630 Hario Megatsari https://orcid.org/0000-0001-6555-319X

Trias Mahmudiono https://orcid.org/0000-0002-3128-2173

Wantanee Kriengsinyos https://orcid.org/0000-0001-82625095

\section{REFERENCES}

1. Ali R. Non-communicable diseases. Paper presented at: 4th International Conference on Public Health; 2018 Jul 19-21; Bangkok, Thailand.

2. Center of Statistic of East Java. Number of population and growth rate of population by region/city in East Java Province, 2010, 2014, and 2015. Jawa Timur: Center of Statistic of East Java; 2015 (Indonesian).

3. Indonesia Ministry of Health. Health service and improvement for elderly; 2015 [cited 2019 Jul 20]. Available from: http:// www.depkes.go.id/ (Indonesian).

4. International Diabetes Federation. Diabetes prevalence: Thailand [cited 2018 Jul 22]. Available from: https://www.idf.org/ our-network/regions-members/western-pacific/members/ 115-thailand.html.

5. Kurniawan I. Type 2 diabetes mellitus among elderly. Indones Med Assoc 2010;60(12):582 (Indonesian).

6. Indonesia Ministry of Health. Report of Indonesia basic health survey 2013 [cited 2019 Jul 20]. Available from: http://www. depkes.go.id/resources/download/general/Hasil\%20Riskesdas\%202013.pdf (Indonesian).

7. Wild $S H$, Byrne $C D$. $A B C$ of obesity. Risk factors for diabetes and coronary heart disease. BMJ 2006;333(7576):1009-1011.

8. Chao $M$, Wang C, Dong X, Ding M. The effects of tai chi on type 2 diabetes mellitus: a meta-analysis. J Diabetes Res 2018;2018: 7350567.

9. Bandura A. Self-efficacy in changing societies. New York: Cam- 
bridge University Press; 2009, p. 307-311.

10. Centers for Disease Control and Prevention. Physical activity basics: how much physical activity do you need? [cited 2018 Jul 22]. Available from: https://www.cdc.gov/physicalactivity/ basics/adding-pa/barriers.html.

11. Amati F, Dubé JJ, Coen PM, Stefanovic-Racic M, Toledo FG, Goodpaster BH. Physical inactivity and obesity underlie the insulin resistance of aging. Diabetes Care 2009;32(8):15471549.

12. Bull FC, Maslin TS, Armstrong T. Global physical activity questionnaire (GPAQ): nine country reliability and validity study. J Phys Act Health 2009;6(6):790-804.

13. World Health Organization. Global recommendations on physical activity for health; 2010 [cited 2019 Jan 23]. Available from: https://www.who.int/dietphysicalactivity/factsheet_recommendations/en/.

14. Indonesia Ministry of Labor. Normal retirement age and the age of maximum retirement for participants in the pension fund regulation. Jakarta: Indonesia Ministry of Labor; 1995 (Indonesian).

15. Scavini M, Stidley CA, Shah VO, Narva AS, Tentori F, Kessler DS, et al. Prevalence of diabetes is higher among female than male Zuni indians. Diabetes Care 2003;26(1):55-60.

16. Alice JayaPradha Cheekurthy AJ, Rambabu C, Kumar A. Prevalence of type 2 diabetes mellitus among women and the associated risk factors. Res Rev J Nurs Health Sci 2016;2(2):26-30.

17. Kautzky-Willer A, Harreiter J, Pacini G. Sex and gender differences in risk, pathophysiology and complications of type $2 \mathrm{di}-$ abetes mellitus. Endocr Rev 2016;37(3):278-316.

18. Aru WS, Bambang S, Idrus A, Marcellus SK, Siti S. Textbook of internal medicine; vol. 3. 54th ed. Jakarta: Interna Publishing; 2015, p. 1857-1858 (Indonesian).

19. Koopman RJ, Mainous AG 3rd, Diaz VA, Geesey ME. Changes in age at diagnosis of type 2 diabetes mellitus in the United States, 1988 to 2000. Ann Fam Med 2005;3(1):60-63.

20. Moretto MC, Fontaine AM, Garcia CA, Neri AL, Guariento ME. Association between race, obesity and diabetes in elderly community dwellers: data from the FIBRA study. Cad Saude Publica 2016;32(10):e00081315.

21. DeFronzo RA. Pathogenesis of type 2 diabetes mellitus. Med
Clin North Am 2004;88(4):787-835.

22. Meneilly GS, Tessier D. Diabetes in elderly adults. J Gerontol A Biol Sci Med Sci 2001;56(1):M5-M13.

23. Knowler WC, Barrett-Connor E, Fowler SE, Hamman RF, Lachin $J M$, Walker EA, et al. Reduction in the incidence of type 2 diabetes with lifestyle intervention or metformin. N Engl J Med 2002;346(6):393-403.

24. McCarthy MM, Davey J, Wackers FJ, Chyun DA. Predictors of physical inactivity in men and women with type 2 diabetes from the Detection of Ischemia in Asymptomatic Diabetics (DIAD) study. Diabetes Educ 2014;40(5):678-687.

25. Wadén J, Tikkanen H, Forsblom C, Fagerudd J, Pettersson-Fernholm K, Lakka T, et al. Leisure time physical activity is associated with poor glycemic control in type 1 diabetic women: the FinnDiane study. Diabetes Care 2005;28(4):777-782.

26. Hu G, Jousilahti P, Barengo NC, Qiao Q, Lakka TA, Tuomilehto J. Physical activity, cardiovascular risk factors, and mortality among Finnish adults with diabetes. Diabetes Care 2005;28(4): 799-805.

27. Mora S, Cook N, Buring JE, Ridker PM, Lee IM. Physical activity and reduced risk of cardiovascular events: potential mediating mechanisms. Circulation 2007;116(19):2110-2118.

28. Katzmarzyk PT, Lee IM. Sedentary behaviour and life expectancy in the USA: a cause-deleted life table analysis. BMJ Open 2012;2(4):e000828.

29. Allison MJ, Keller C. Self-efficacy intervention effect on physical activity in older adults. West J Nurs Res 2004;26(1):31-46.

30. Gray PM, Murphy MH, Gallagher AM, Simpson EE. Motives and barriers to physical activity among older adults of different socioeconomic status. J Aging Phys Act 2016;24(3):419429.

31. Guicciardi M, Lecis R, Anziani C, Corgiolu L, Porru A, Pusceddu $M$, et al. Type 2 diabetes mellitus, physical activity, exercise self-efficacy, and body satisfaction. An application of the transtheoretical model in older adults. Health Psychol Behav Med 2014;2(1):748-758.

32. Bethancourt HJ, Rosenberg DE, Beatty T, Arterburn DE. Barriers to and facilitators of physical activity program use among older adults. Clin Med Res 2014;12(1-2):10-20. 